\title{
ABSTRACT KOROVKIN THEORY FOR DOUBLE SEQUENCES VIA POWER SERIES METHOD IN MODULAR SPACES
}

\author{
FADIME DiRIK, SEVDA YILDIZ AND KAMIL DEMIRCI
}

Abstract. In the present paper, we obtain an abstract version of the Korovkin type approximation theorems for double sequences of positive linear operators on modular spaces in the sense of power series method. We present an example that satisfies our theorem but not satisfies the classical one and also, we study an extension to non-positive operators.

Mathematics subject classification (2010): 40G10, 41A36, 46E30.

Keywords and phrases: Power series method, double sequence, abstract Korovkin theorem.

\section{REFERENCES}

[1] G. A. Anastassiou And O. Duman, Towards intelligent modeling: Statistical approximation theory, Intelligent System Reference Library, Springer-Verlag, Berlin, Heidelberg, New York, 2011.

[2] C. Bardaro, A. Boccuto, K. Demirci, I. Mantellini, S. Orhan, Korovkin-type theorems for modular $\Psi-A$-statistical convergence, J. Funct. Spaces, 2015, Article ID 160401, 2015, pp. 11.

[3] C. Bardaro, A. Boccuto, X. Dimitriou and I. Mantellini, Abstract Korovkin type theorems in modular spaces and applications, Cent. Eur. J. Math., 11, 10, 2013, 1774-1784.

[4] C. BARDARO AND I. MANTEllini, Korovkin's theorem in modular spaces, Commentationes Math., 47, 2, 2007, 239-253.

[5] C. Bardaro, J. Musielak, G. Vinti, Nonlinear integral operators and applications, de Gruyter Series in Nonlinear Analysis and Appl., Vol., 9, Walter de Gruyter Publ., Berlin, 2003.

[6] C. Bardaro AND I. Mantellini, A Korovkin Theorem in multivariate modular function spaces, J. Funct. Spaces Appl., 7, 2, 2009, 105-120.

[7] S. BARON, U. STADTMÜLlER, Tauberian theorems for power series methods applied to double sequences, J. Math. Anal. Appl., 211, 2, 1997, 574-589.

[8] A. Boccuto AND X. Dimitriou, Korovkin-type theorems for abstract modular convergence, Results in Mathematics, 69, 3-4, 2016, 477-495.

[9] S. Karakuş, K. Demirci, O. Duman, Statistical approximation by positive linear operators on modular spaces, Positivity, 14, 2, 2010, 321-334.

[10] P. P. Korov kin, Linear Operators and Approximation Theory, Hindustan Publ. Co., Delhi, 1960.

[11] W. M. Kozlows KI, Modular function spaces, Pure Appl. Math., Vol. 122, Marcel Dekker, Inc., New York, 1988.

[12] K. Kuratows Ki, Topology, Volls I and II, Academic Press, New York-London, 1966/1968.

[13] I. Mantellini, Generalized sampling operators in modular spaces, Commentationes Math., 38, 1, 1998, 77-92.

[14] J. MusielaK, Orlicz spaces and modular spaces, Lecture Notes in Mathematics, Vol. 1034 SpringerVerlag, Berlin, 1983.

[15] J. MUSIELAK, Nonlinear approximation in some modular function spaces I, Math. Japon., 38, 1, 1993, $83-90$.

[16] S. ORhan, K. Demirci, Statistical approximation by double sequences of positive linear operators on modular spaces, Positivity, 19, 1, 2015, 23-36.

[17] A. Pringsheim, Zur Theorie der zweifach unendlichen Zahlenfolgen, Math. Ann., 53, 1, 1900, 289 321. 